\title{
Congenital Seminal Vesicle Cyst and Ipsilateral Renal Agenesis (Zinner Syndrome): A Rare Association and Its Evolution from Early Childhood to Adolescence
}

\author{
Aikaterini Kanavaki $^{1} \quad$ Isabelle Vidal $^{2}$ Laura Merlini ${ }^{1} \quad$ Sylviane Hanquinet ${ }^{1}$ \\ ${ }^{1}$ Department of Pediatric Radiology, Geneva's Children University \\ Hospital, Geneva, Switzerland \\ 2 Department of Pediatric Surgery, Geneva's Children University \\ Address for correspondence Aikaterini Kanavaki, MD, Department of \\ Pediatric Radiology, Geneva's Children University Hospital, Rue Willy- \\ Donzé 6, Geneva 1205, Switzerland (e-mail: katerinakan@yahoo.gr).
} Hospital, Geneva, Switzerland

Eur J Pediatr Surg Rep 2015;3:98-102.
Abstract
Keywords
- seminal vesicle
- cyst
- renal agenesis
- Zinner syndrome

Zinner syndrome, the association of congenital seminal vesicle cyst and ipsilateral renal agenesis, is more often reported in adults or older adolescents. We present a case of a boy, followed up in our hospital since birth for right renal agenesis who at the age of 4 years presented a right paravesical cyst on ultrasound. The cyst was initially considered as an ureterocele. The diagnosis of Zinner syndrome was made later, at the age of 15 years by ultrasound and magnetic resonance imaging; at that moment the cyst had increased in size and had changed in aspect. This malformation should be considered in the differential diagnosis of a pelvic cyst in male patients with renal agenesis.

\section{New Insights and the Importance for the Pediatric Surgeon}

Congenital seminal vesicle cysts in patients with ipsilateral renal agenesis are rare but this association is well known. Pediatric surgeons should be aware and consider this entity in the differential diagnosis of cystic pelvic masses in males with renal agenesis or dysplasia. Ultrasound is useful for diagnosis but magnetic resonance imaging provides a more detailed analysis and accurate diagnosis. Treatment is considered only for symptomatic patients and is surgical. Follow-up is proposed for asymptomatic or minimally symptomatic cysts.

\section{Introduction}

Congenital seminal vesicle cysts associated with abnormalities of the upper urinary tract are uncommon. ${ }^{1}$ They can be asymptomatic and discovered incidentally or can be associated with dysuria, urinary tract infections, and infertility. Diagnosis is more frequently made in adult life during the period of the greatest sexual or reproductive activity. ${ }^{1,2} \mathrm{We}$ present a case of a congenital seminal vesicle cyst associated with ipsilateral renal agenesis incidentally discovered on ultrasound at the age of 4 years and its natural evolution

received

November 28, 2014

accepted after revision

April 30, 2015

published online

July 10, 2015 until adolescence. The association of congenital seminal vesicle cyst with ipsilateral renal malformations is rare and was first described by Zinner in 1914. It is reported in literature as "Zinner syndrome."” This condition is considered as the male equivalent of Mayer-Rokitansky-Kustner-Hauser (MRKH) syndrome described in females. ${ }^{4}$

\section{Case Report}

A 4-year-old boy with known right renal agenesis discovered on antenatal ultrasound, presented in our radiology

\footnotetext{
(c) 2016 Georg Thieme Verlag KG Stuttgart · New York
} 
department for his annual control. A retrovesical cyst ipsilateral to the renal agenesis was detected on ultrasound (-Fig. 1a). The cyst was anechoic, measured $8 \mathrm{~mm}$, and was not present on previous ultrasounds. It was initially considered as an ureterocele or bladder diverticula, however, neither of these diagnosis was confirmed on intravenous urography performed at that time. The patient was asymptomatic. On regular ultrasonographic controls, the cyst remained unchanged on size and aspect during 11 years of follow-up. However, at the age of 15 years its aspect on the ultrasound had changed: its content was hyperechoic, it had increased in size (measuring $1.9 \mathrm{~cm}$ ), and protruded in the bladder. It was associated to magma of some echogenic, round retrovesical masses, initially considered as lymph nodes ( - Fig. 1 b, c). Magnetic resonance imaging (MRI) of the urinary tract and pelvis was further performed (coronal, axial, and sagittal T2 spin-echo and axial T1 with fat saturation before and after gadolinium injection images). It revealed a dilatation of the seminal vesicles, ipsilateral to renal agenesis (corresponding to the magma of retrovesical round masses seen on ultrasound), ending to a relatively small seminal vesicle cyst (diameter of $\sim 2 \mathrm{~cm}$ ) that protruded in the bladder (-Fig. 2 a-c).

So far, the patient was asymptomatic and had never presented with dysuria, signs of bladder obstruction or urinary tract infection. He had neither any perineal discomfort nor pain, he described normal ejaculation without pain, no hematospermia, and had never experimented any urinary infection or prostatitis and also he was not sexually active.

In this particular case, in the absence of symptoms and because of the relatively small size of the cyst a conservative treatment was decided, with an annual clinical and ultrasonographic control, at least until the first symptoms appear.

\section{Discussion}

The association of congenital seminal vesicle cyst with ipsilateral renal malformations is rare and there are approximately 200 cases reported in the literature. ${ }^{4}$

This association is explained by the common embryologic origin of both the organs (kidneys-seminal vesicles) from the mesonephric duct ${ }^{1}$ and is due to an insult in embryogenesis between the 4th and 13th week of gestation. ${ }^{4}$ The ureteral bud arises from the dorsal part of the distal mesonephric duct and extends dorsocranially to meet and induce differentiation of the metanephric blastema, from which the kidney will develop. The mesonephric duct will differentiate to epididymis, ejaculatory duct, vas deferens, seminal vesicle, and hemitrigone. Complete failure of the mesonephric duct results to absence of ipsilateral kidney, ureter, hemitrigone, and seminal vesicle. Anomalous development of the distal mesonephric duct results to atresia of the ejaculatory ducts and abnormal ureteral budding; the former leads to obstruction and cystic dilatation of the seminal vesicles with development of seminal cysts, the latter leads to renal agenesis or dysplasia. ${ }^{2,5}$ Zinner syndrome could be considered as the male equivalent of MRKH syndrome described in females. ${ }^{4}$
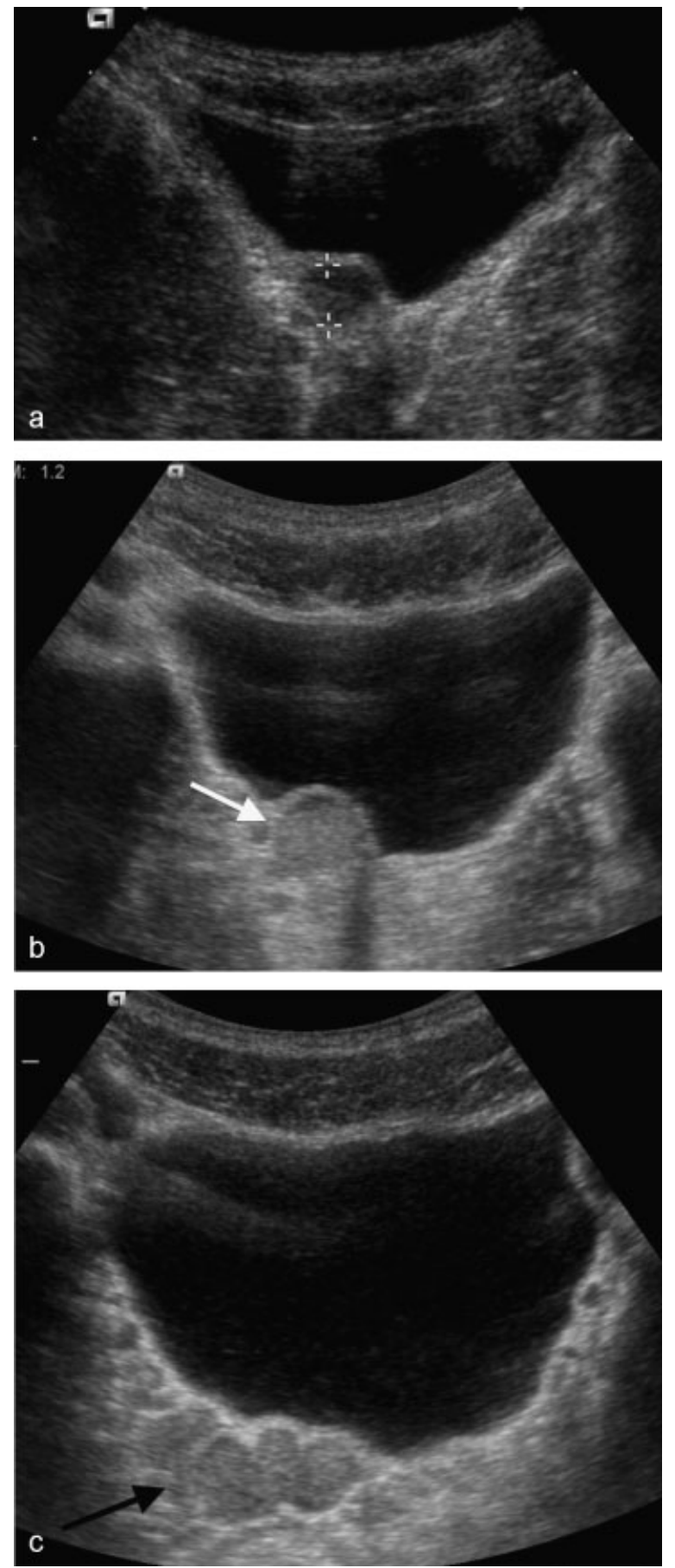

Fig. 1 Ultrasound (a) at the age of 4 years detects a right retrovesical anechoic cyst $(+)$ (b) at the age of 15 years the cyst has increased in size and it has become hyperechoic (white arrow). (c) It is associated to magma of round retrovesical masses (black arrow).

The cysts are present since birth but enlarge and become symptomatic on late adolescence or adult life, usually in the third to fifth decade, at the period of greatest reproductive or sexual activity. ${ }^{1,2}$ At this moment the accumulation of secretions in the seminal vesicle due to atresia of the ejaculatory ducts and consequently insufficient drainage leads to formation of cysts in the seminal vesicle. ${ }^{2}$

Cysts smaller than $5 \mathrm{~cm}$ are usually asymptomatic and are discovered incidentally. Larger cysts can irritate the 

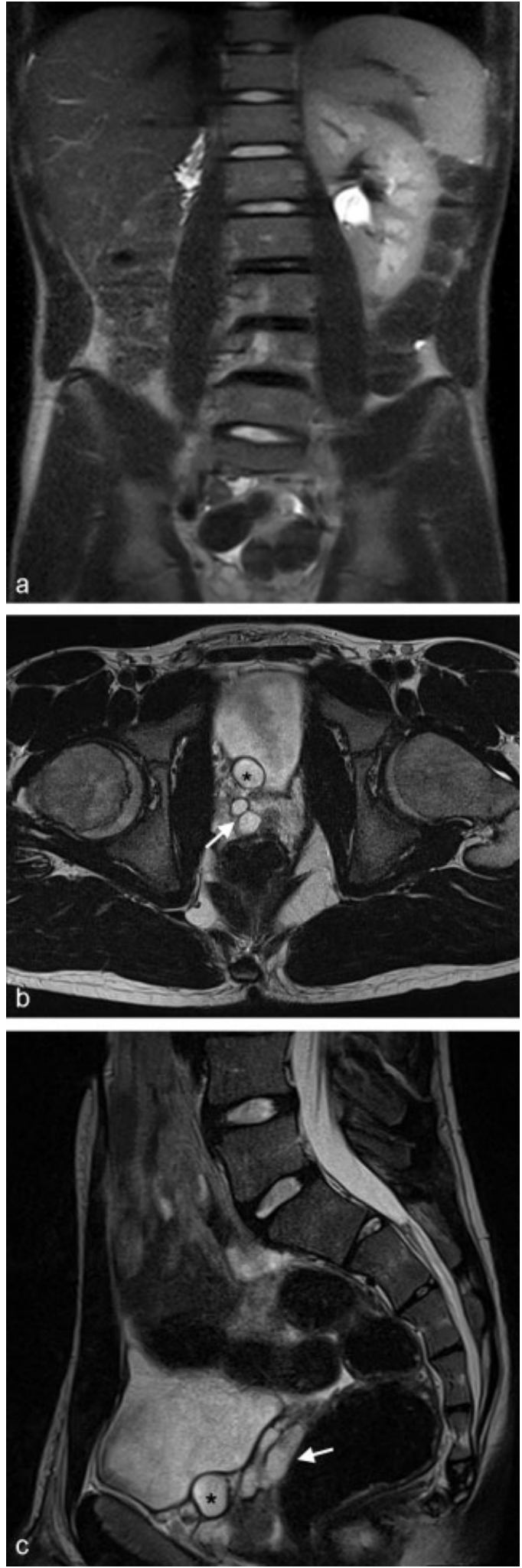

Fig. 2 MRI at the age of 15 years. (a) T2 coronal images of the abdomen show right renal agenesis. (b) T2 spin-echo axial and (c) sagittal images of the pelvis show dilatation of the right seminal vesicles (white arrow) ending to a seminal vesicle cyst $\left(^{*}\right)$ that protrudes in the bladder. MRI, magnetic resonance imaging.

bladder and be related to pain, dysuria, frequency, hematuria, urinary tract infection, epididymitis or prostatitis, infertility, hemospermia, bladder outlet, or colonic obstruc- tion. ${ }^{1,2,6}$ Malignant degeneration of the cysts has been also reported. ${ }^{2,4}$

This progressive dilatation of seminal vesicle remains often asymptomatic until the dilatation increases to 4 to $5 \mathrm{~cm}, 4,5$ unless the obstruction triggers vas deferens dilatation at younger age, ${ }^{5}$ which was not the case for our adolescent. This dilatation develops slowly, by accumulation of secretions in the seminal vesicle, for many years after puberty, due to congenital atresia of the ejaculatory ducts and consequently insufficient drainage ${ }^{2}$ : literature reports a majority of cases becoming symptomatic around the third or fourth decade of life. Diagnosis can also be made earlier in front of infertility linked to contralateral distal ejaculatory pathway compression by this cystic seminal vesicle. ${ }^{3}$

In our case, the cyst was first seen at the age of 4 years, mimicking an ureterocele on ultrasound. In adolescence it increased in size, but remained small and, for this reason, asymptomatic. Diagnosis was made on MRI, based on its anatomic connection and to the similar signal intensity with the seminal vesicles.

Differential diagnosis includes other pelvic cystic masses such as utricular or Mullerian cyst, ureterocele, dilated ureter, abscess or lymph nodes, ${ }^{2,4}$ and acquired seminal vesicle cysts. Acquired cysts are usually bilateral and concern older patients with chronic prostatitis or postprostate surgery.

Ultrasound raises the suspicion of this malformation but the final diagnosis is usually made by MRI (or computed tomography, with this last method being irradiating). In adults more invasive methods as cystoscopy and vasovesiculography are also used for diagnosis. ${ }^{2}$

There is a medical consensus to propose conservative follow-up for asymptomatic or minimally symptomatic cysts. $^{1,4,7}$ Treatment is considered only for symptomatic patients and is surgical. Different surgical options exist, from the less invasive transrectal or transperineal aspiration of the cyst which gives a transitory relief of the symptoms, ${ }^{1}$ to more aggressive technics. Transurethral unroofing of the cyst by transurethral resection of the ejaculatory duct provides improvement of the quality of semen and improves the paternity rate, ${ }^{3}$ but this procedure can create injury to rectum, bladder neck, and external sphincter, and induce consequent retrograde ejaculation, and epididymitis.

Radical treatment, vesiculectomy with resection of ureteral or renal remnants if present, offers the better outcome. It was traditionally performed by open exploration but is now well described laparoscopically, with low morbidity and good results in term of symptoms relief and semen parameters. ${ }^{1,4,7-11}$ Few children with surgical indication benefited from this approach. ${ }^{12,13}$

Concerning fertility, for patients presenting with difficulty to procreate, with low volume of semen and poor quality of spermogram, treatment shows semen quality improvement, and paternity obtained without other medical help., ${ }^{3,4}$ Assisted reproductive procedures should be kept for patients with infertility persisting after successful surgical procedure. ${ }^{4}$

Our case is original as it shows the natural history and evolution of this malformation, from childhood to 
adolescence. Cases reported in literature concern, in their high majority the adult population (or late adolescence) and these cysts are rarely detected at a younger age.

\section{Conclusion}

Congenital seminal vesicle cysts in patients with ipsilateral renal agenesis are rare but this association is known and should be considered in the differential diagnosis of cystic pelvic masses in males with renal agenesis or dysplasia. Ultrasound is useful for diagnosis but MRI provides a more detailed analysis and accurate diagnosis. Treatment is considered only for symptomatic patients and is surgical. Follow-up is proposed for asymptomatic or minimally symptomatic cysts.

\section{References}

1 Kao CC, Wu CJ, Sun GH, et al. Congenital seminal vesicle cyst associated with ipsilateral renal agenesis mimicking bladder outlet obstruction: a case report and review of the literature. Kaohsiung J Med Sci 2010;26(1):30-34

2 Livingston L, Larsen CR. Seminal vesicle cyst with ipsilateral renal agenesis. AJR Am J Roentgenol 2000;175(1):177-180

3 Pace G, Galatioto GP, Gualà L, Ranieri G, Vicentini C. Ejaculatory duct obstruction caused by a right giant seminal vesicle with an ipsilateral upper urinary tract agenesia: an embryologic malformation. Fertil Steril 2008;89(2):390-394
4 Pereira BJ, Sousa L, Azinhais P, et al. Zinner's syndrome: an up-todate review of the literature based on a clinical case. Andrologia 2009;41(5):322-330

5 Ghonge NP, Aggarwal B, Sahu AK. Zinner syndrome: A unique triad of mesonephric duct abnormalities as an unusual cause of urinary symptoms in late adolescence. Indian J Urol 2010;26(3):444-447

6 Sundar R, Sundar G. Zinner syndrome: an uncommon cause of painful ejaculation. BMJ Case Rep 2015;2015(15):xx

7 Cherullo EE, Meraney AM, Bernstein LH, Einstein DM, Thomas AJ, Gill IS. Laparoscopic management of congenital seminal vesicle cysts associated with ipsilateral renal agenesis. J Urol 2002;167(3): 1263-1267

8 Liatsikos EN, Lee B, Filos KS, Barbalias GA. Congenital seminal vesicle cyst and coexisting renal agenesis: laparoscopic approach. Urology 2004;63(3):584-586

9 Seo IY, Kim HS, Rim JS. Congenital seminal vesicle cyst associated with ipsilateral renal agenesis. Yonsei Med J 2009;50(4):560-563

10 van den Ouden D, Blom JH, Bangma C, de Spiegeleer AH. Diagnosis and management of seminal vesicle cysts associated with ipsilateral renal agenesis: a pooled analysis of 52 cases. Eur Urol 1998; 33(5):433-440

11 Jarzemski P, Listopadzki S, Kowalski M. Laparoscopic removal of a congenital seminal vesicle cyst in Zinner's syndrome. JSLS 2014; 18(2):367-371

12 Basillote JB, Shanberg AM, Woo D, Perer E, Rajpoot D, Clayman RV. Laparoscopic excision of a seminal vesicle cyst in a child. J Urol 2004;171(1):369-371

13 Valla JS, Carfagna L, Tursini S, Mohaidi MA, Bosson N, Steyaert H. Congenital seminal vesicle cyst: prenatal diagnosis and postnatal laparoscopic excision with an attempt to preserve fertility. BJU Int 2003;91(9):891-892 